Ann. Génét. Sél. anim., I969, 1 (4), 403-4I2.

\title{
PROGRÈS DES CONNAISSANCES GÉNÉTIQUES SUR L'INTERSEXUALITÉ ASSOCIÉE A L'ABSENCE DE CORNES CHEZ LA GHÈVRE D'ORIGINE ALPINE
}

\author{
J.-J. LAUVERGNE \\ Station centrale de Génétique animale, \\ Centre national de Recherches zootechniques, 78-Jouy-en-Josas \\ Institut national de la Recherche agronomique
}

RÉSUMÉ

La connaissance génétique de l'intersexualité associée à l'absence de cornes chez la Chèvre domestique a été lentement acquise. Cette lenteur est due à la multiplicité, maintenant pleinement reconnue, des actions du mutant autosomal $P$ qui, à l'état homozygote, masculinise toutes les femelles en transformant la plupart en intersexués et certaines en pseudo-mâles, d'où les déséquilibres du sex-ratio en faveur des mâles, même après avoir compté les intersexués avec les femelles. Une certaine confusion est née également du fait que ce facteur agit aussi chez les mâles homozygotes pour déclencher une autre forme de stérilité. A côté de son action stérilisante, le gène induit également une action superfécondante dans les deux sexes. Au total treize paramètres sont nécessaires pour caractériser l'action de ce facteur.

\section{INTRODUCTION}

L'originalité de l'étude génétique de l'intersexualité associée à l'absence de cornes chez la chèvre domestique réside dans une pléiotropie aux multiples facettes. Ce n'est que graduellement que s'est fait jour la vision selon laquelle toute une série de phénomènes constamment associés dans les lignées de chèvres sans cornes originaires des Alpes et plus précisément de Suisse, pouvaient avoir une origine génétique factorielle unique : intersexualité, différentes formes de stérilité des mâles, décalage đu sex-ratio en faveur des mâles, surfécondité de certaines femelles. 
Les trois jalons principaux dans le cheminement des conceptions génétiques nous semblent être les travaux d'AsderL (r944, b) de BRANDSch (I959) et de SorLER et al. (I969). Le premier de ces auteurs a fait la liaison entre l'absence de cornes et 1'intersexualité, le second y a rattaché le phénomène de renversement du sexe phénotypique pour expliquer les écarts du sex-ratio, les troisièmes ont démontré, quant à eux, que l'action stérilisante du facteur n'était pas limitée, comme on l'avait cru longtemps au sexe féminin.

\section{A. - LA GENÈSE DE L'HYPOTHÈSE D'ASDELL (1944, b)}

I1 serait intéressant de préciser les modalités exactes de la migration du caractère sans cornes à partir des souches suisses dans les lignées améliorées pour lesquelles s'ouvrent, dès la fin du siècle dernier, des livres généalogiques un peu partout en Europe et aux États-Unis. Avec la pression de la mode, la rapidité de ce phénomène a pu être très grande.

Dans ces lignées le caractère motte $\left(^{1}\right)$ a été étudié génétiquement par ASDEILI. et CREW (I925) et par LUSH (I926), il s'est révélé monofactoriel autosomal dominant dans les deux sexes.

Quant à l'intersexualité, signalée déjà à la fin du siècle mais surtout à partir des années vingt, elle a été étudiée pour la première fois par CREW (I923), puis par Funkouist (I932) et EATon et Simmons (I939). Ces derniers auteurs la considéraient comme monofactorielle récessive alors que FuNKQUIST songeait à un gène situé sur $\mathrm{X}$. Déjà rendues difficiles par la variation d'expressivité du caractère, ces études n'ont pu se baser sur le déterminisme du sexe génétique des intersexués difficilement envisageable à l'époque aussi bien directement (caryolologie) qu'indirectement (statistique).

L'idée selon laquelle absence de cornes et intersexualité ont un déterminisme factoriel associé s'est fait jour au début des années quarante et 1'on dispose de témoignages permettant d'en suivre toutes les étapes, qui se résument à peu près comme suit.

Tout a commencé par une controverse provoquée en Angleterre par un article d'ASDELI (I942) selon lequel on devait assister à une disparition, vite totale, de tous les sujets cornus dans les lignées de chèvres dès le moment où l'on écartait de la reproduction tous les animaux sans cornes. Or, comme le fait remarquer un des lecteurs du British Goat Society Monthly Journal dans une lettre à l'éditeur parue peu après (HoLMEs, I942) cela n'est pas. En I932, en effet, a été entrepris un programme d'éradication des reproducteurs cornus dans le Herd-Book alpin de Grande-Bretagne et en I94I on observe encore 40 p. Ioo de chevreaux cornus. Un peu plus tard, toujours préoccupé par ces questions de cornage et d'intersexualité, un autre lecteur du même journal, $\operatorname{PAGET}(I 943$, a) lançait une enquête auprès des éleveurs afin de recueillir tous renseignements concernant le sexe et le cornage des parents comme des enfants.

L'analyse des données de son enquête a amené PAGET (I943, b) à se poser la question suivante : pourquoi n'y-a-t-il que des intersexués sans cornes? L'année

(1) En français " motte " signifie * sans cornes ", c'est l'équivalent de "polled " en anglais. 
suivante AsDELL (I944, a) propose une explication : le gène dominant pour l'absence de cornes serait étroitement lié au gène récessif d'intersexualité. Mais cela n'est pas tout et cet auteur, prenant conscience qu'il existe un déficit considérable des femelles dans les troupeaux sans cornes, pense que les intersexués sont des femelles génétiques XX (ASDELL. I944, b).

L'hypothèse d'ASDELL parlait de deux gènes étroitement liés; EATON (I945), un peu plus tard fit remarquer qu'il suffisait d'invoquer un seul gène pléiotropique, opinion partagée par L,USH dans une lettre à ASDELL (I946) et qui semble définitivement admissible puisque, depuis le temps, on n'a jamais pu séparer des souches présentant seulement de l'intersexualité de souches sans cornes. A partir de cette époque, ce gène autosomal s'est appelé $P, p$ désignant 1'allèle normal.

\section{B. - LES INSUFFISANCES DE L'HYPOTHËSE D'ASDELL, LA REMARQUE DE BUECHI (I957) E'T L'HYPOTHÈSE DE BRANDSCH (1959)}

L'hypothèse d'ASDELL ( $\left.{ }^{\mathbf{1}}\right)$ ne précisait pas la pénétrance de l'action d'intersexualité chez les homozygotes, implicitement les auteurs l'ont considéré comme égale à roo p. Ioo et c'est sur cette base que EAToN (I945) sur les données du troupeau de Beltsville et un peu plus tard, Kondo au Japon (I952, I955) ont cru vérifier cette proposition.

Vers cette époque, cependant, commençaient à s'accumuler, en Allemagne surtout, des travaux consacrés à la stérilité de certains boucs mottes. Commencées depuis longtemps dans ce pays, ces études ont eu, au début des années cinquante, un renouveau de faveur (par exemple : SchöNHERR, I954 : LöLIGER, I957).

C'est le décalage du sex-ratio, phénomène perçu certainement moins généralement que l'infertilité des mâles qui est à l'origine de la première remise en cause par BuEchi (I957) en Suisse de l'hypothèse d'AsDex.L. Analysant les données des Herd-books sans cornes de son pays, cet auteur décelait en effet un excès notable et hautement significatif du nombre des mâles par rapport à la fréquence de $5^{\circ} \mathrm{p}$. roo. Selon lui, 1'hypothèse basée sur le fait qu'on devait retrouver un sexratio normal en ajoutant les intersexués aux femelles était alors inacceptable.

Toutefois, 1'explication suggérée par BuEchi selon laquelle le décalage du rapport des sexes est dû à un excès de mortalité des femelles in utero ne permet pas de rattacher ce phénomène à l'action du gène responsable, de 1'intersexualité et de l'absence de cornes dont il reconnaît, par ailleurs, 1'unicité. Les bases statistiques d'une telle explication restent fragiles tant par la modicité des effectifs examinés que par le fait qu'on ne peut affirmer que le gène d'intersexualité n'est pas capable de masculiniser très tôt des fœtus génétiquement femelles.

S'appuyant sur des données statistiques nombreuses et sur les importants travaux allemands sur la stérilité des mâles dans les races sans cornes, BRANDSCH (I959) va introduire une clause qui permettra de conserver, pour l'essentiel, les

(1) Que certains appellent l'hypothèse d'EAToN à cause de la contribution apportée par cet auteur. Il nous semble, quant à nous, difficile de passer sous silence lcs mérites d'AsDell. Il faudrait au moins dire l'hypothèse d'ASDELL-EATON. 
vues d'ASDELL. Le raisonnement de ce nouvel auteur est simple et ne va pas sans rappeler celui d'ASDEL, concernant le sexe des intersexués : les mâles stériles, constamment présents dans les troupeaux sans cornes, sont en réalité des pseudomâles, femelles génétiques très masculinisées, il suffit de les ajouter aux femelles vraies et aux intersexués pour rétablir le sex-ratio normal. Les fréquences génotypiques dans les croisements entre hétérozygotes vérifient en outre, l'hypothèse de pénétrance totale de l'action masculinisante chez les femelles homozygotes $P P\left({ }^{1}\right)$ puisqu'on observe $37,67 \mathrm{p}$. Ioo de femelles à la naissance pour un chiffre de 37,5 p. Ioo. L'auteur pense pouvoir, en outre, déterminer l'expressivité de chaque forme : $50 \mathrm{p}$. Ioo de pseudo-mâles, $50 \mathrm{p}$. Ioo d'intersexués parmi les homozygotes $P P$ génétiquement femelles.

\section{C. - DE BRANDSCH (1959) A SOLLER ET AL. (I969)}

Même en l'absence d'une détermination cytologique du sexe génétique des anormaux qui n'était d'ailleurs pas encore envisageable pratiquement à l'époque, les éléments d'une critique permettant de rectifier l'hypothèse de BRANDSCH étaient déjà réunis au début des années 60 . Cela n'a pas été fait et on a même assisté à un oubli de ces résultats qui ont dû être retrouvés un à un non sans une certaine confusion. en même temps que se développaient des moyens plus précis d'une détermination du sexe génétique par la caryologie. Essayons de dégager tour à tour ces aspects : critique rétrospective des conceptions de BRANDSch, redécouverte des résultats déjà acquis et progrès.

\section{Critique rétrospective des conceptions de BRANDSCH}

BRANDSCH considère en bloc la stérilité des mâles, or, il ressort de l'examen des articles allemands déjà évoqués qu'il y a, en fait, deux catégories de mâles stériles : ceux qui présentent des granuloma au niveau de l'épididyme avec blocage du transit des spermatozoïdes (sténose) et ceux qui présentent une hypoplasie des testicules. Les premiers peuvent parfois être fertiles lorsque l'atteinte est unilatérale ou bénigne, les seconds sont toujours stériles. Par ailleurs, une étude biométrique de LöHLE et BARFuss (I96I) a montré que ces deux types se distinguent par leurs mensurations, les premiers se rapprochant du type mâle, les seconds du type femelle. Ainsi, il semble bien difficile d'assimiler la catégorie des mâles sténosiques à des femelles génétiques entièrement masculinisées. Il aurait fallu alors que l'on observe des sex-ratios de roo p. roo de femelles dans la descendance de ceux, qui, par hasard, étaient fertiles, ce qui n'a jamais été rapporté. Un autre obstacle à cette assimilation de tous les mâles stériles à des femelles génétiques masculinisées réside dans les statistiques. SchöNHERR (I954) nous indique en effet qu'en race allemande blanche sans cornes on observe I9,5 p. Ioo de mâles stériles parmi les boucs mottes. Ce chiffre se rapproche fort des $20 \mathrm{p}$. Ioo

(1) C'est-à-dire leur disparition totale en tant que reproduction et la seule existence d'hétérozygotes parmi les femelles mottes. 
de mâles stériles prévisibles si tous les reproducteurs mâles de la population étaient homozygotes. Or, cette condition est loin d'être remplie puisque BRANDSCH (I959) dans une autre race il est vrai. mais avec la même sélection en faveur des animaux sans cornes, détecte seulement II p. Ioo de mâles homozygotes. En outre, la fréquence des mâles stériles dans une population est certainement donnée par défaut car on ne compte pas les animaux dont 1'hypoplasie a été tôt détectée et qui ont disparu précocement. Aussi doit-on conclure que le nombre de mâles stériles observés dépasse largement celui qui est attendu. Cette contradiction peut être levée en supposant que tous les mâles stériles ne sont pas des femelles génétiques, c'est-à-dire en envisageant également une action stérilisante du gène $P$ dans le sexe mâle.

\section{2. - Redécouverte des anciens résultats et acquisitions nouvelles}

A partir de 1962, les Israéliens publient leurs travaux sur l'intersexualité associée à l'absence de cornes chez la Chèvre (LAOR et al., I962, SoLLER et al. I963, Solier et ANGel I964, Soller et KeMPenich I964). Leurs conceptions sont résumées dans l'article présenté à la Conférence des Éleveurs de Chèvres de Londres I964 (LAOR et al., I964).

Le principal mérite des Israéliens à cette époque est d'avoir mis en évidence l'existence d'une stérilité frappant $80 \mathrm{p}$. Ioo des mâles homozygotes $P P$, confirmant ainsi l'hypothèse qu'il était, nous l'avons vu un peu plus haut, déjà possible d'émettre à l'époque. Quant à l'effet du gène sur le sex-ratio, ils se montrent moins catégoriques que BRANDSCH pour l'attribuer au changement phénotypique de certaines femelles. D'une manière générale, ils attendent, pour conclure avec certitude, les examens caryologiques qui déjà à l'époque ont confirmé que le sexe génétique des intersexués était bien femelle (cf. HuLOT et BASRUR, I969) et qui doivent apporter la lumière sur le sexe génétique des mâles stériles. Avec Sol, LER et al (1969) les premières preuves que les mâles hypoplasiques sont des femelles et les mâles à épididyme bloquée sont des mâles sont apportées, la question est résolue.

Chemin faisant, les Israéliens avaient mis en évidence une propriété encore inconnue du gène $P$, celle d'augmenter de $5 \mathrm{p}$. Ioo la fécondité des femelles hétérozygotes $P p$ par rapport aux homozygotes cornues $(p p)$. Comme pour la stérilité, cette action sur la fécondité ne semble pas limitée au sexe femelle puisque RICORDEAU et LAUVERGNE ( 1967 ) détectaient déjà une surfécondité des mâles homozygotes $P P$ par rapport aux hétérozygotes et surtout aux homozygotes, phénomène dont la réalité semble prouvée par les derniers résultats donnés par RICORDEAU (I969).

\section{D. - TABLEAU ACTUEL DU COMPORTEMENT DU FACTEUR $P$}

\section{A TRAVERS SEXES ET GÉNOTYPES}

Si l'on reprend maintenant en détail les différents aspects du comportement du gène $P$, selon les génotypes et les sexes, on voit qu'il faut, pour les décrire, employer I3 paramètres dont la signification est résumée dans le tableau I et dont l'estimation est proposée dans le tableau 2. 
TABLEAU I

Modes d'action du gène $P$ selon les sexes et les génotypes

\begin{tabular}{|c|c|c|c|c|}
\hline \multirow{2}{*}{$\begin{array}{l}\text { Sexe } \\
\text { géné- } \\
\text { tique }\end{array}$} & \multirow{2}{*}{$\begin{array}{l}\text { Formule } \\
\text { chromoso- } \\
\text { mique }\end{array}$} & \multicolumn{3}{|c|}{ Génotypes } \\
\hline & & $p p$ & $P p$ & $P P$ \\
\hline dర0 & $\mathrm{XY}$ & Mâles cornus fertiles & $\begin{array}{l}I-a\left({ }^{1}\right) \text { mâles mottes fer- } \\
\text { tiles plus prolifiques que } \\
\text { les mâles } p p\left(r_{1}>I\right) \\
a \text { mâles mottes atteints } \\
\text { d'obstruction de l'épidi- } \\
\text { dyme : } \\
x_{1} \text { partiellement (restent } \\
\text { fertiles) } \\
x_{2} \text { d'une manière généra- } \\
\text { lisé (stériles) }\end{array}$ & $\begin{array}{l}I-b \text { mâles mottes fertiles } \\
\text { plus prolifiques que les } \\
\text { mâles } p p\left(r_{2}>r_{1}>I\right) \text {. } \\
b \text { mâles mottes atteints } \\
\text { d'obstruction de l'épi- } \\
\text { didyme : } \\
\beta_{1} \text { partiellement (restent } \\
\text { fertiles) } \\
\beta_{2} \text { d'une manière géné- } \\
\text { lisée (stériles) }\end{array}$ \\
\hline 유 & $\mathrm{xx}$ & Femelles cornues fertiles & $\begin{array}{l}\text { Femelles mottes fertiles } \\
\text { plus prolifiques que les } \\
\text { femelles } p p(t>\mathrm{I})\end{array}$ & $\begin{array}{l}\text { Animaux mottes tous } \\
\text { stériles }(c=1): \\
\gamma_{1} \text { pseudo-mâles } \\
\gamma_{2} \text { intersexués } \\
\gamma_{3} \text { femelles stériles }\end{array}$ \\
\hline
\end{tabular}

(I) $a, b, c, \alpha_{1}, \alpha_{2}, \beta_{1}, \beta_{2}, \gamma_{1}, \gamma_{2}$ et $\gamma_{3}$ sont des proportions, leur valeur est comprise entre o et $\mathrm{I}$.

Trois sont des paramètres de pénétrance : $a$ et $b$ pour les atteintes épididymaires chez, respectivement, les hétérozygotes et les homozygotes mâles sans cornes, $c$ pour la stérilité chez les homozygotes femelles sans cornes. Trois sont des coefficients de sélection différentielle : $r_{1}$ et $r_{2}$ pour tenir compte de la surfécondité des mâles $P P$ et $P p$ par rapport aux $p p, t$ pour tenir compte de la surfécondité des femelles $P p$ par rapport aux $p p$.

Sept sont des expressivités : $\alpha_{1}$ et $\alpha_{2}$ et $\beta_{1}$ et $\beta_{2}$ des 2 formes partielle (avec maintien de la fertilité) et généralisée (accompagnée de stérilité) d'atteintes épididymaires chez, respectivement, les hétérozygotes et les homozygotes mâles sans cornes; $\gamma_{1}, \gamma_{2}$ et $\gamma_{3}$ des différentes anomalies présentées par les femelles homozygotes $P P$.

La situation est un peu simplifiée car deux valeurs de pénétrances apparaissent sinon nulle, du moins fort proche de la nullité : celle des anomalies épididymaires chez les mâles hétérozygotes : $a=0$ (les Israéliens ne découvrent aucun mâle $P p$ stérile, SOLLER et al. I963) et celle de la stérilité chez les femelles $P P$ où elle serait totale : $c=I$ (RICORDEAU et LAUVERGNE, I967). De cette manière $\alpha_{1}$ et $\alpha_{2}$ ne présenteraient plus d'intérêt, il resterait à évaluer $b, \beta_{1}, \beta_{2}, \gamma_{1}, \gamma_{1}, \gamma_{3}$, $r_{1}$, $\gamma_{2}$, et $t$ en se rappelant que $\beta_{1}+\beta_{2}=\mathrm{I}$ et que $\gamma_{1}+\gamma_{2}+\gamma_{3}=\mathrm{I}$. 
TABLEAU 2

Estimation des paramètres qui caractérisent l'action du gène $P$

\begin{tabular}{|c|c|c|c|c|}
\hline \multicolumn{2}{|r|}{ Paramètres } & \multicolumn{3}{|c|}{ Estimées } \\
\hline Sigle & Définition & Valeur & Données fournies par & Remarques \\
\hline$a$ & $\begin{array}{l}\text { Pénétrance des atteintes } \\
\text { épididymaires chez les } \\
\text { mâles } P p\end{array}$ & o & SOLLER et al. 1963 . & \\
\hline$b$ & $\begin{array}{l}\text { Pénétrance des atteintes } \\
\text { épididymaires chez les } \\
\text { mâles } P P\end{array}$ & 0,912 & $\begin{array}{l}\text { SCHÖNHERR (I954) } \\
+ \text { BRANDSCH (I959). }\end{array}$ & cf. LAUVERGNE (I969). \\
\hline$c$ & $\begin{array}{l}\text { Pénétrance de la stérilité } \\
\text { chez les femelles } P P \text {. }\end{array}$ & $\begin{array}{l}\text { I } \\
\text { I }\end{array}$ & $\begin{array}{l}\text { BRANDSCH, I959. } \\
\text { RICORDEAU et LAUVER- } \\
\text { GNE, I967. }\end{array}$ & \\
\hline$\alpha_{1}$ & $\begin{array}{l}\text { Expressivité des attein-- } \\
\text { tes unilatérales chez les } \\
\text { mâles } P P \text { atteints de sté- } \\
\text { nose épididymaire }\end{array}$ & & & sans intérêt si $a=0$. \\
\hline$\alpha_{2}$ & $\begin{array}{l}\text { Expressivité des atteir- } \\
\text { tes bilatérales chez les } \\
\text { mâles } P p \text { atteints de sté- } \\
\text { nose épididymaire }\end{array}$ & & & sans intérêt si $a=0$. \\
\hline$\beta_{1}$ & $\begin{array}{l}\text { Expressivité des attein- } \\
\text { tes unilatérales chez les } \\
\text { mâles } P P \text { atteints de sté- } \\
\text { nose épididymaire }\end{array}$ & $\begin{array}{l}0,638 \\
0,43\end{array}$ & $\frac{\text { SCHÖNHERR (I954). }}{\text { WEBER (I969). }}$ & $\begin{array}{l}\text { Estimations statistique- } \\
\text { ment différentes. }\end{array}$ \\
\hline$\beta_{2}$ & $\begin{array}{l}\text { Expressivités des attein- } \\
\text { tes bilatérales chez les } \\
\text { mâles } P P \text { atteints de sté- } \\
\text { nose épididymaire }\end{array}$ & $\begin{array}{l}0,362 \\
0,57\end{array}$ & $\frac{\text { SCHÖNHERR (I954). }}{\text { WEBER (I969). }}$ & $\begin{array}{l}\text { Estimations statistique- } \\
\text { ment différentes. }\end{array}$ \\
\hline$\alpha_{1}$ & $\begin{array}{l}\text { Expressivité de la forme } \\
\text { "pseudo-mâle ", chez } \\
\text { les femelles } P P \text {. }\end{array}$ & $\begin{array}{l}0,56 \\
0,43\end{array}$ & $\frac{\text { BRANDSCH (I959). }}{\text { BUECHI (I957). }}$ & $\begin{array}{l}\text { Estimations non signifi- } \\
\text { cativement différentes. }\end{array}$ \\
\hline$\gamma_{2}$ & $\begin{array}{l}\text { Expressivité de la forme } \\
\text { "intersexué " chez les } \\
\text { femelles } P P \text {. }\end{array}$ & $\begin{array}{l}0,44 \\
0,57\end{array}$ & $\frac{\text { BRANDSCH (I959). }}{\text { BUECHI (I957) }}$ & $\begin{array}{l}\text { Estimations non signifi- } \\
\text { cativement différentes. }\end{array}$ \\
\hline
\end{tabular}


TABLEAU 2 (suite)

Estimation des paramètres qui caractérisent l'action du gène $P$

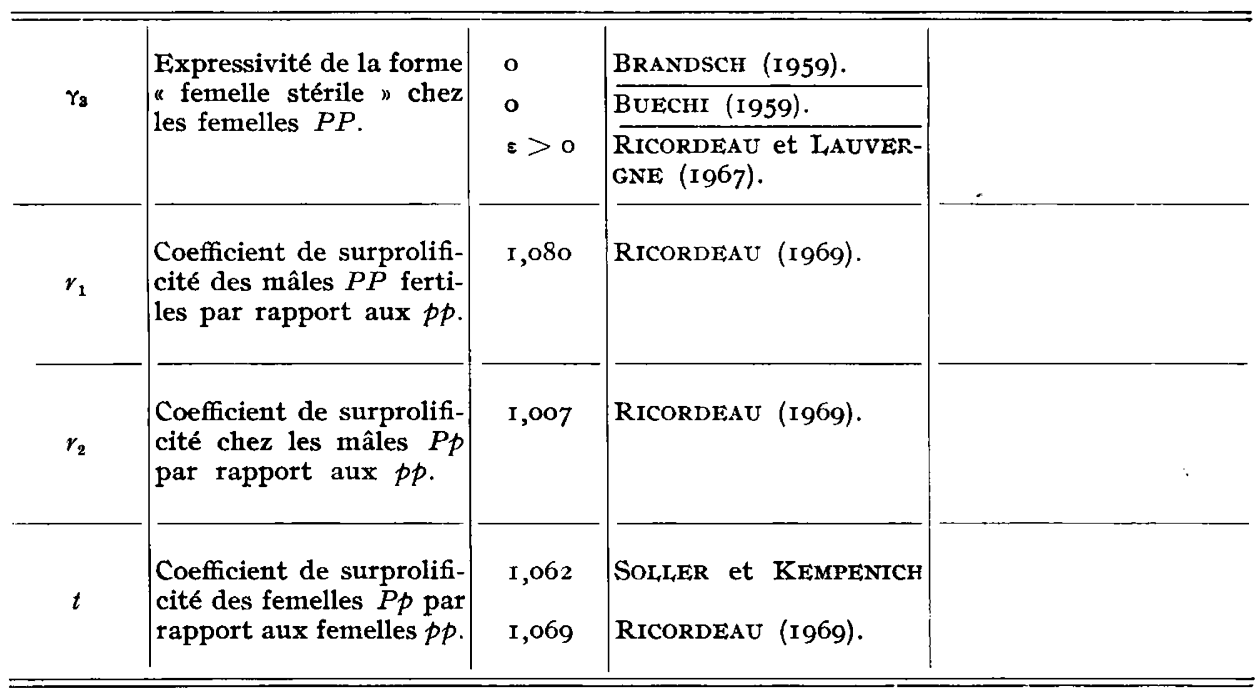

L'estimation de $b$ à partir des données de SchöNHERR (I954) est possible si l'on connaît la fréquence des homozygotes $P P$ chez les jeunes mâles. Anticipant sur la discussion de génétique des populations (LAUVERGNE, I969) nous pouvons prendre pour la fréquence gamétique mâle de $P$ le chiffre déterminé en Allemagne à partir des données de BRANDSCH (I959) : 0,5544. On a alors parmi les jeunes mâles une fréquence de $\frac{0,5444^{2}}{0,5+0,5544^{2}}$ pour les homozygotes $P P$. Faisant abstraction des animaux hypoplasiques (qui sont des pseudo-mâles) dans le tableau 9 de ScHöNHERR on a 97 I mâles dont 366 homozygotes qui se répartissent quant à leur fertilité en 32 normaux, 213 " acceptables sous conditions " et 121 " inacceptables ". Si l'on assimile les catégories " acceptables sous conditions " et " inacceptables moins hypoplasiques" de ScHöNHERR respectivement à nos formes " atteintes épididymaires partielles " et " atteintes épididymaires généralisées " on obtient pour $b$ la valeur 0,912 , pour $\beta_{1}: 0,638$ et pour $\beta_{2}: 0,362$.

WEBER (I969) nous fournit pour $\beta$, et $\beta_{2}$ une autre estimation en distinguant quant à lui sténosiques uni- et bilatéraux. Les estimées fournies par SchöNHERR (r954) et WEBER (r969) sont significativement différentes. Cette différence s'explique peut-être par des différences dans l'intensité de la sélection à l'encontre des sténosiques homozygotes unilatéraux qui, rappelons-le, sont fertiles. Il n'est pas impossible non plus que $b$ puisse varier sous l'influence de cette même sélection.

Pour $\gamma_{1}$ et $\gamma_{2}$ que l'on peut estimer à partir des données de BuEchr (1967) et BRANDSCH (I959) en comptant le nombre des mâles stériles comme étant les mâles en surnombre et en se plaçant dans l'hypothèse où $\gamma_{3}=0$ (pas de femelles stériles), les valeurs ne sont pas significativement différentes. Quant à $r_{1}$ et $r_{2}$, ils ont été récemment estimés par RICORDEAU (I969). 


\section{CONCLUSIONS}

Les premiers travaux remontant à $\mathbf{1 9 2 5}$, on peut constater que les progrès de la connaissance génétique de l'intersexualité associée à l'absence de cornes ont été fort lents. Cela tient tout d'abord au coût et à la lenteur des recherches sur une espèce de Ruminants comme la Chèvre. Cela tient, en deuxième lieu à l'extrême originalité de la pléiotropie de ce facteur autosomal. Toutefois, bien que le but final semblait se đérober à mesure que l'on progressait, il faut remarquer que les hypothèses successives de ASDELL puis de BRANDSCH ne contenaient pas de propositions fondamentalement fausses mais demandaient simplement des précisions pour des phénomènes qui, à peine discernés par les auteurs, pouvaient difficilement entrer dans des schémas présentant déjà une grande nouveauté pour leur époque.

Pour l'heure toutefois, il semble que l'on soit parvenu à une vue générale qui ne changera guère et qui demande seulement des précisions. Ces précisions pourront être fournies par deux voies : $\mathrm{r}^{0}$ ) La génétique des populations, à partir des données des sociétés d'élevage qui, tout en permettant de vérifier les hypothèses dans des lignées où un type de sélection bien défini s'est exercé, devrait nous fournir des estimations de certaines pénétrances et expressivités. $2^{\circ}$ ) Les croisements expérimentaux en stations qui donneront des renseignements plus précis mais seront plus coûteux à mettre en place.

Rę̧u poù publication en octobre 1969.

\section{SUMMARY}

PROGRESS IN GENETIC KNOWLEDGE OF INTERSEXUALITY ASSOCIATED WITH POLLEDNESS IN THE GOAT OF AIPINE ORIGIN

It is useful to distinguish three principal stages in the genetic studies of intersexuality linked with the polled condition in alpine goats. The landmarks for these stages are the works of Asdell $(1944, b)$, Brandsch (1959) and Soller et al. (1959).

AsDell $(1944, b)$ tried to show that intersexuality restricted to females was of the autosomal recessive type influenced by a neighbouring gene which was dominant for the absence of horns, and that the intersexes were generally $\mathrm{XX}$ females. In the absence of crossing over it is wise to consider, as did EAToN (1945) and Lush (in Aspell, 1946) that it is a question of one and the same gene.

The hypothesis of AsDell, does not permit, as shown by BuEchi (1957) an explanation for the constant excess of males in the sex ratio, even if one counts the intersexes as females. BRANDsch's work (1959) placed the sterile males (whose existence in herds of polled goats has been noted for some time by German authors) in a genetic scheme based on the assumption that sterile males were actually strongly masculinized females $(P P)$ for the polled trait. However, this explanation did not take into account the existence of two categories of sterile males: Those affected by testicular hypoplasia and those with granulomas in the epididymus which blocked the seminiferous ducts.

The Israelis can take credit for suggesting that certain males were genetically male (SoLLER et al., 1963) and for cytologically proving this point (Soller et al., 1969). They also showed that those sterile males with testicular hypoplasia were genetically females as had been partially forseen by BRANDSCH.

In order to complete the statement on the phenotypic expression of the gene $P$ (summarized in Table 1) it is necessary to mention a superprolificity of hornless heterozygous females $P p$ in comparison to horned homozygotes (Soller and KEMPENICH, 1964) and of homozygotes males PP with $P p$ and $p p$ (Ricordeau and Lauvergne, 1967; Ricordeau, 1969).

Thirteen parameters have to be considered to measure all the functional aspects of the gene $P$ across sexes and genotypes; a certain number of these are known $(a, c)$ while the others are approximations (cf. Table 2). 


\section{RÉFÉRENCES BIBLIOGRAPHIQUES}

AsDell S.A., 1942. Goat research in 1941. Yb. Br. Goat Soc., 50-51.

Asdell S.A., 1944, a. Hermaphrodites. Mon. J. Goat. Soc., 37, 13-15.

AsDell S.A., 1944 b. The genetic sex of intersexual goats and a probable linkage with the gene for hornless. ness. Science, N.Y., 99, 124 .

ASDELl S.A., 1946. Goat research in r944-45. Yb. Br. Goat Soc., 29-32.

AsDell S.A., Crew F.A.E., 1925. The inheritance of horns in the Goat. J. Genet., 15, 367-374.

BRANDSCH H., 1959. Die Vererbung geschlechtlicher Missbildung und des Hornes bei der Hausziege in ihrer gegenseitigen Beziehung. Arch. Geflügelzucht Kleintierkde., 8, 310-362.

BUEchI H.F., I957. Untersuchungen über das verschobene Geschlechtsverhältnis, die Intersexualität und die Fruchtbarkeit bei der Milchziege. Z. Tierzücht. ZüchtBiol., 69, 30-9o.

CREW F.A.E., I932. Studies in intersexuality. I.A peculiar type of developmental intersexuality in the male of the domesticated Mammals. Proc. r. Soc. London, 95, 90-rog.

Earon O.N., 1945. Relation between polled and hermaphroditic characters in dairy Goat. Genetics, Princeton, 30, 5r-6r.

Eaton O.N., Srmmons V.I., I939. Hermaphrodism in milk Goats. J. Hered., 30, 26I-266.

Funkouist H., 1932. Über Vererbung von Hypospadia bei Ziegen. Biologia Gen., 8, 59-64.

Holmes W.F., 1942. Iettre à l'éditeur. Mon. J. Brit. Goat Soc., 35, 122.

Hulot Françoise, BASRUR, Parvathi K., I969. La détermination du sexe chromosomique dans l'étude de l'intersexualité associée à l'absence de cornes chez la Chèvre d'origine alpire. Ann. Génét., Sél. anim., 1, 383-390.

KoNDO K., 1952. Studies on intersexuality in milk Goats. Jap. J. Genet., 27, I31-141.

KoNDo K., 1955. The frequency of occurence of intersexes in Milkgoats. Jap. J. Genet., 30, I39-146.

I.AOr, M., Barnea R., ANgel Huguette, Ayalon N., Weise I., Soller M., I964. Polledness and hermaphroditism, male sterility, sex-ratios and litter size in Israel Saanen Goats. Int. Goat Breeders conf., London, July 1964 (ronéoté).

I Aor M., Barnea R., ANgel H., Soller M., r962. Polledness and hermaphroditism in Saanen Goats. Israel J. agric. Res., 12, 83-88.

LAUVERGne J.-J., I969. Fréquences à l'équilibre du gène d'origine alpine responsable de l'absence de cornes et de l'intersexualité dans les populations de Chèvres. Ann. Génét. Sél. Anim., 1, 4r3-422.

IöHLE K., BARFUSS E., I96r. Untersuchungen über Korpergewichte und Korpermasse bei fruchtbaren und unfruchtbaren Ziegenböcken. Arch. Geflügelzucht. Kleintierkde., 10, 121-128.

LöLIGER H.C., I957. Die Verbreitung von Unfruchtbarkeit unter den männlichen Tieren verschiedener Ziegenrasse in deutschen und ausländischen Zuchtgebieten. Zuchthyg. Fortpflstör Besam. Haustiere, 1, $201-215$.

LUSH J.-I., r926. Inheritance of horns, wattles and color in grade Toggenburg Goats. J. Hered., 17, 73-9r.

PAGet R.F., I943 a. I,ettre à l'éditeur. Mon. J. Brit. Goat. Soc., 36, I7.

PAGET R.F., 1943 b. Hermaphrodites. Mon. J. Brit. Goat Soc., 36, 57-59.

RICORDEAU G., I969. Surprolificité des génotypes sans cornes dans les races caprines alpines Saanen, alpine chamoisée et poitevine. Ann. Génét. Sél. Anim., 1, 391-395.

Ricordeau G., LAUVERGNE J.J., 1967. Hypothèse génétique unique pour expliquer la présence d'intersexués, de mâles en excès et de mâles stériles en race caprine Saanen. Ann. Zootech., 16, 323-334.

SchöNherR S., 1954. Die Unfruchtbarkeit der Ziegenböcke, ihre Verbreitung, früheitige Erkennung und Bekämpfung. Vet. Med., Diss. Frei Universität. Berlin.

Soller M., ANgel H., 1964. Polledness and abnormal sex ratios in Saanen Goats. J. Heved, 55, I39-142. Soller M., Kempenich O., I964. Polledness and litter size in Saanen Goats. J. Hered., 55, $301-304$.

Soller M., LAOr M., Barnea R., Weiss Y., Ayalon N., r963. Polledness and infertility in male Saanen Goats. J. Hered., 54, 237-240.

Soller M., Padeh B., Wysoki M., Ayalon N., 1969. Cytogenetics of Saanen Goats showing abnormal development of the reproductive tract associated with the dominant gene for polledness. Cytogenetics, 8, $51-67$.

WVeber W., r969. La stérilité des mâles dans trois races caprines suisse s.uns cornes. Ann. Génét. Sél. Anim., 1, 379-382. 